\title{
Digital twin: its concept and potential application in the coal industry
}

\author{
Denis Korovin ${ }^{1}{ }^{*}$, Vitaliy Grachev ${ }^{1}$, Georgiy Kulyushin ${ }^{1}$, and Danil Ivanov ${ }^{2}$ \\ ${ }^{1}$ Siberian State Industrial University, Novokuznetsk, 654007, Russia \\ ${ }^{2}$ Research Center of Control Systems LLC, Novokuznetsk, 654005, Russia
}

\begin{abstract}
The article is devoted to the specific character of using digital twin technology in the coal industry. The key points and nuances of this technology application in the industry in general and in the coal industry in particular are discussed.
\end{abstract}

\section{Introduction}

The phrase "digital twins" that has become fashionable nowadays requires a deeper understanding and linking with many years of achievements in the construction and application of models (modeling), in complex natural-mathematical models that combine both mathematical and natural components, methods and control systems with a physical model, the systemic principles of the synthesis of control systems with statements of the fundamental impossibility to build a universal model, with a reflection of behavior in human-machine systems. It is also necessary to study more closely the experience of using mathematical models in various industries. Otherwise, unbridled optimism from digital twins will lead to disappointment, as has happened more than once in other spheres of theory and practice. The presented material of the article is formed from these positions.

\section{Relevance}

Coal mining is the leading economic activity of the Kemerovo Region. The level of development of the coal mining industry largely determines the level of development of the economy of the entire region. The volumes of coal production have a long-term positive trend, which requires even greater dynamics of the development of the processing and coal preparation industry. The strategy of the socio-economic development of the Kemerovo Region until 2035 determines that one of the promising directions for the development of the industry is an increase in the processing of extracted raw materials. The increase in the total processing volumes predetermines the need for both the modernization of existing coal preparation complexes and the creation of new enterprises.

At the moment, the degree of digitalization of the mining industry is quite high automated control systems have been introduced, including operational control and longterm storage of controlled parameters of technological processes and equipment [1-3]. This

\footnotetext{
* Corresponding author: mail@,nicsu.ru
} 
can be the basis for the creation of digital shadows of enterprises, which, in turn, serves as a "low threshold for entry" into the digital economy of the region and the country as a whole. The next stage of integration is the creation and use of full-scale digital twins of industries. For some industries, digital twins of standard objects have been developed and debugged. It is necessary to develop general structures and specific recommendations for the creation and implementation of full-scale digital twins of mining enterprises.

\section{Concept of "digital twin"}

A digital twin is a virtual, software analogue of a physical product, a group of products or a process that simulates internal processes, technical characteristics and behavior of a real object in conditions of interference and the environment. Its work also consists in collection and reuse of digital information [4].

There is another interpretation of the digital twin. It is a virtual model, which at the micro and macro levels either describes an existing object (acting as a duplicate of a finished product), or serves as a prototype of a future object. Moreover, any information that can be obtained when testing a physical object must also be obtained on the basis of testing its digital twin.

\subsection{Formation of the "Digital twin" concept}

Before the start of the first industrial revolution, which began in the $18^{\text {th }}$ century, various products were mainly made by artisans - the production of unique products by a single template. However, with the introduction of the concept of interchangeable parts in the $18^{\text {th }}$ century, the way of products manufacturing quickly changed as companies began to strive to create large volumes of copies of their products. More recently, the mass personification paradigm has sought to combine these well-established manufacturing concepts to achieve low unit costs for individual products.

However, while such manufacturing paradigms allow the production of many similar parts or products, these instances are simply unrelated copies. By contrast, the idea of creating a twin refers to making a copy of a part or product as a whole and using it to describe other copies - thus establishing a link between multiple copies. This idea arose from the NASA Apollo program, "where at least two identical spacecraft were built to allow a mirror image of the state of the spacecraft during flight" [5-6]. Figure 1 shows the implementation of the twin in the Apollo project.

Briefly tracing the history of the development of the digital twins concept, we can say that from the moment a person began to create material products, he used virtual twin models. At first, he created them in his imagination, then on paper, then in a computer, and now in the "cloud", using "smart" sensors, the Internet of things, artificial intelligence. At each new stage, new digital technologies and methods of modeling, forecasting, analysis, training were added to the concept.

Over the past decades, advances in computer technology have allowed the creation of increasingly complex virtual models of physical objects, as well as the integration of such models for system design. These models not only serve to validate design, but are increasingly used as a reference model for a product. Moreover, the development of microchips, sensors and information technology has led to the emergence of smart products that track and transmit external parameters and, thus, allow the model to be "filled' with data on the state of the product, such as environmental conditions and external influences on the product [7-8]. 


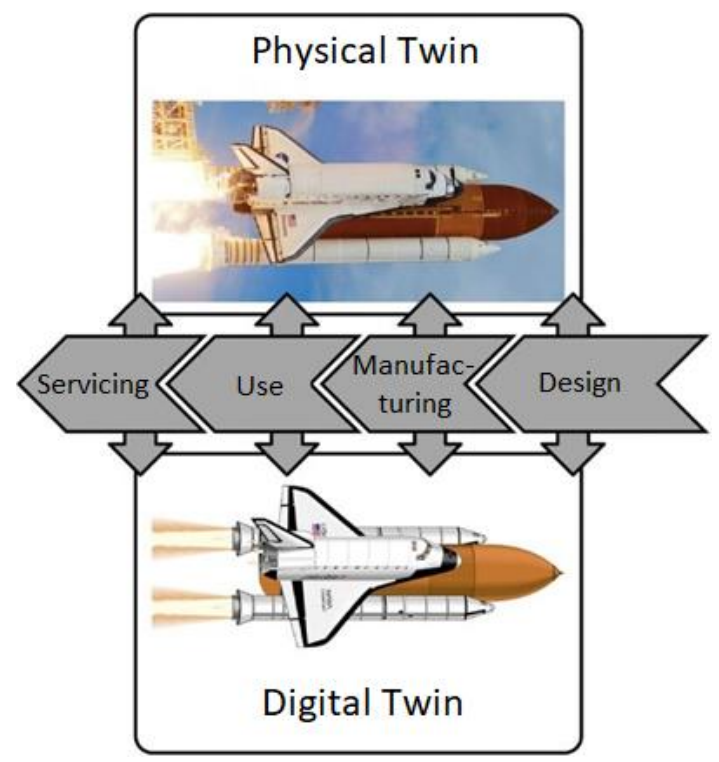

Fig. 1. The vision of a twin throughout the product life cycle in the Apollo project.

The digital twin is based on a real model, which means that the twin must take all readings from real sensors. The general structure of the digital twin technology is shown in Fig. 2.



Fig. 2. General structure of the "digital twin" technology.

By receiving readings from the sensors, it is possible to set the input disturbances to the twin. Taking this into account, the readings of real sensors with those of the digital twin can be compared. Based on these readings, the conclusions about the disagreements and the reasons for their occurrence are drawn.

The digital twin contains:

- digital model;

- material characteristics;

- manuals and data for the maintenance of the product;

- information about the influence of external factors.

The most effective application of digital twin technology is for products with the following criteria:

- product support by qualified specialized service (condition control, monitoring, technical support);

- long product life cycle (from 5 to 70 years);

- a large number of copies of installed equipment; 
- a wide range and variety of operating conditions;

- inaccessibility of the product for maintenance.

This is a very extensive list of criteria for products from various industries: technological units of coal mines and processing plants, aircraft engines and systems, complex industrial equipment, railway and automobile transport systems, medical equipment [9-12].

\subsection{Classification of digital twins}

At the moment, digital twins are being used in many industries, such as the coal industry, energy, and mechanical engineering. The main reason for using digital twins in these industries is the cost of error. In each of these industries, an operator's, dispatcher's or an engineer's mistake can lead to both economic damage and a threat to human life and health.

According to the widespread classification there are types of twins: digital twin prototypes (Digital Twin Prototype, DTP), digital twin instances (Digital Twin Instance, DTI), and aggregated twins (Digital Twin Aggregate, DTA).

A DTP twin characterizes the physical object being its prototype and contains the information needed to describe and create a physical version of the object. This information includes manufacturing requirements, an annotated $3 \mathrm{D}$ model, bill of materials, processes, services, and disposal.

DTI twins describe a specific physical entity with which the twin remains associated throughout its life. Twins of this type usually contain an annotated 3D model with general dimensions and tolerances, a bill of materials that lists current and past components, a bill of materials listing the operations that were performed when this physical object was created, and the results of any tests of the object, service records, including replacement of components, operating indicators, test and measurement results obtained from sensors, current and predicted values of monitoring parameters.

DTA twins are defined as a computing system that has access to all digital twins and can send random or proactive queries to them.

The symbiosis with the technologies of the Internet of Things is a driver of the development of digital twin technologies. Digital twins receive real data from sensors that monitor real objects, while the Internet of Things ensures the collection and analysis of data from various types of sensors and makes this process economical and efficient.

\section{Methods of digital twin implementation}

Digital twins of manufacturing enterprises and their processes are a comprehensive tool for planning, optimization, finding vulnerabilities and improving overall control efficiency. In a general sense, digital twins are created for already existing objects and rely on their information display, various types of models and methods of mathematical analysis. At the same time, the choice of the most effective of them for each new object is possible only based on the results of comparison after numerous experiments - that is, on the fact of already committed mistakes.

For some industries, digital twins of standard objects have been developed and debugged. However, it is impossible to completely transfer a ready-made debugged system to a new object, since even seemingly insignificant differences can cause significant deviations and errors. Every time a significant revision is required even for similar productions. In the first case, it is known that a reasonable transfer of control decisions is possible only if the control systems are similar. That is, it is necessary to develop the theory of similarity of control systems from the conditions of functioning of digital twins [13]. Such solutions can be carried over from a previously developed system, or from a physical 
(laboratory or semi-industrial) model. As a rule, physical or semi-industrial models are first developed to study technology and operating modes in new industries. The methods and grounds for transferring the obtained results of processes research are described by the theory of physical processes similarity. The development of the theory of similarity for control systems, firstly, made it possible to substantiate the transfer of a part of the control system by linking the properties of models of control objects, external influences, regulation laws, and, secondly, take into account the systemic features caused by the closure of direct and reverse control connections.

In the second case, it is advisable to apply the methods of full-scale mathematical modeling using typical representative situations that develop the general concept of the fullscale model approach worked out in the scientific school of control systems modeling. Since these methods satisfy the restrictions on the volume and quality of a priori information inherent in full-scale operating systems of control automation, in particular, the lack of adequate mathematical models "in the big"; limited possibilities for conducting active experiments at objects in the modes of their operation; minimal information about the properties of various kinds of disturbances; unforeseen possible changes in management objectives; replacement of raw materials components; modernization of the control objects during operation, etc.

In general, the vision of a digital twin describes the vision of a bidirectional relationship between a physical object and its many virtual models. In this context, the establishment of relationships between physical parts and their virtual models allows design, production, service and various other activities to be effectively carried out throughout the entire life cycle of the product (production). In particular, when designing such digital twins, it is possible to check the compliance of the technical characteristics of the product with the general concept and requirements of the customer.

However, as reported in the scientific literature and in practice, the current limitations of the digital twin implementation are insufficient synchronization capabilities between the physical and digital world to establish closed loops, the lack of high-precision models for modeling and virtual testing at several scales, the lack of a quantitative estimate of the uncertainty for such models, the difficulties of forecasting complex systems, as well as the problems of collecting and processing large amounts of data. Indeed, these problems can only be solved on the basis of a solid conceptual framework and a comprehensive digital twin reference model.

A full-scale digital twin is understood as a full-scale model complex integrated into the full-scale (or physical) control system, displaying its functioning in digital form.

\section{Conclusion}

The creation of new and improvement of existing automation systems for the control of production facilities implies, among other things, an increase in the level of digitalization for their integration into the modern environment of the digital economy. Digital twins are one of the main tools for this. However, at the moment, the scientific and practical foundations for the creation of common structures of both digital twins and complex fullscale control automation systems, their concretization in production, educational and scientific activities, have been poorly developed. When solving this problem, it is advisable to focus on the natural-model approach, the effectiveness of which has been confirmed by many years of theoretical and practical testing in the automation of control in the mining and metallurgical industries.

Almost all researchers agree on the purpose of digital twins - to increase the efficiency of a business or production. For this, the main emphasis is placed on the material flows of 
the entire enterprise, without revealing the essence of the technological processes and management processes occurring within local systems and circuits.

Digital twins can be a useful tool. They improve maintenance operations and simplify product support, save money, reduce failures, and extend equipment life. A digital twin can serve as a source of field data for further research, without the need for access to a real object, since access to a real object is often difficult for reasons of security of the object or its territorial remoteness.

\section{References}

1. L.P. Myshlyaev, Automation systems based on a natural-model approach: Monograph in 3 volumes (Nauka, Novosibirsk, 2006)

2. G.P. Sazykin, B.A. Sineokiy, L.P. Myshlyaev, Design and construction of new generation coal preparation plants (SibSIU, Novokuznetsk, 2003)

3. V.V. Grachev, L.P. Myshlyaev, D.E. Korovin, G.A. Kulyushin, The use of BIM technologies in the design of automated industrial complexes, in Procedings of the conference on Automated electric drive and industrial electronics in the metallurgical and mining and fuel industries, 86-94 (2020)

4. A. Prokhorov, M. Lysachev, A. Borovkov, Digital twin. Analysis, trends, world experience (AlliancePrint LLC, Moscow, 2020)

5. R. Rosen, G. von Wichert, G. Lo, K.D. Bettenhausen, About the Importance of Autonomy and Digital Twins for the Future of Manufacturing. IFAC-Papers OnLine, 48(3), 567-572 (2015)

6. E.H. Glaessgen, D.S. Stargel, The digital twin paradigm for future NASA and U.S. air force vehicles, Proceedings of the 53rd AIAA Structures, Structural Dynamics and Materials Conference (2012)

7. Digital Twin: prospects for using digital twins https://nfp2b.ru/2019/01/09/digitaltwin-perspektivy-ispolzovaniya-tsifrovyh-dvojnikov/ (2019)

8. L.P. Myshlyaev, K.G. Wenger, V.V. Grachev, Digitalization and management, in Proceedings of the Conference on Automation systems in education, science and production, AS'2019, 29-31 (SibSIU, Novokuznetsk, 2019)

9. V.I. Danilov-Danilyan, Ecology, hydrology, digitalization, digital twins and the elementary truths of modeling methodology, in Proceedings of the conference on Scientific problems of the improvement of Russian rivers and ways to solve them, 497-502 (2019)

10. V.N. Bykova, E. Kim, et al., Actual Problems of Oil and Gas, 8 (2020)

11. N.A. Eremin, A.N. Eremin, Oil. Gas. Novations, 12, 14-17 (2018)

12. D.S. Kokorev, N.P. Posmakov, Colloquium-journal, 26-2(50), 71-78 (2019)

13. G.V. Makarov, K.A. Ivushkin, et al., Evaluation of the similarity of control systems, in Procedings of the conference on Automated electric drive and industrial electronics, 170-177 (2016) 